\section{Cosmic Rays and Mutations}

THE following results have a bearing on the problem touched upon by Dr. Hamshaw Thomas in his paper "Cosmic Rays and the Origin of Species"1.

In 1933 I had the idea of subjecting Drosophila flies to the action of cosmic rays in the stratosphere. However, it was only in 1935 that I was enabled to carry out my plan, and about 300 Drosophila melano. gaster males were entrusted to the crew which made an ascent from Moscow on June 26 in the stratostat U.S.S.R.-I-bis. The crew of the balloon consisted of K. J. Zille, J. G. Prilutzky and Prof. A. B. Verigo. This experiment was carried out with the view of demonstrating the possible effect of cosmic rays on the mutation process. The experimental flies were placed in the gondola where an average temperature of $12^{\circ}-14^{\circ} \mathrm{C}$. was maintained. The ascent lasted from 5.25 a.m. until 8.2 a.m., the balloon attaining an altitude of 15,900 metres.

According to the data of Prof. Verigo, the experimental flies were for two hours under the action of cosmic radiation which in intensity exceeds cosmic radiation at the level of the ground on the average by 100 times. As soon as the flies landed safely they were studied genetically. The usual $C l B$-method for determining the lethal mutation which occurs in the $X$-chromosome of the sperm of initial males was adopted. At the same time we kept a control batch of flies of the same age and stock (counting 20 days from the egg stage, a wild stock of Nalchik, inbred for five generations).

The following results were obtained: in 2,724 chromosomes of the experimental flies, 8 mutations were obtained $(0 \cdot 294 \pm 0 \cdot 104$ per cent $)$, whereas 2,445 chromosomes of the controls gave 10 mutations $(0 \cdot 409 \pm 0 \cdot 129$ per cent $)$.

These negative results compel us to be circumspect in accepting theories which look upon cosmic rays as important factors in organic evolution.

Institute of Experimental Biology,

HEINRIOH FrIESEN. Moscow.

${ }^{1}$ NATURE, 137, 51 (1936).

\section{Photochemical Reaction of Chlorophyll with Ferrous Ions}

A short time ago, J. Weiss ${ }^{1}$ described in NaTurE some experiments on the photochemical bleaching of dyestuffs in presence of ferrous ions, and interpreted the bleaching effects as reduction-oxidation reactions between the excited dyestuff molecules and the ferrous ion, according to the equation

$$
\mathrm{Fe}++\mathrm{HOH}+\left(D_{8} h \nu\right)=\mathrm{Fe}^{+}+++\mathrm{OH}^{-}+
$$$$
\left(D_{8}-\mathrm{H}\right) \quad(1)
$$

As dyestuffs he used brilliant-cresylblue, methyleneblue, thionine, uranine and chlorophyll.

I should like to point out some difficulties encountered if one tries to reproduce Weiss's experiments using chlorophyll as the photochemically active dyestuff.

Ferrous sulphate, it is true, exerts a bleaching action on chlorophyll, both in neutral and sulphuric acid solution, but this reaction is not influenced by irradiation. It is easy to prove this by adding a small quantity of solution of ferrous sulphate to a solution of chlorophyll in methyl alcohol and irradiating with a strong carbon are after thoroughly removing the atmospheric oxygen (say by evacuating the vessel for a sufficiently long time). The irradiation causes no change of colour. The experiment gives the same result also when sulphuric acid is added ${ }^{2}$. But if atmospheric oxygen is not, or not thoroughly, re. moved, a rapid bleaching of the chlorophyll may be observed, and the solution takes an olive brown coloration, indicating that an oxidation product of chlorophyll has been formed ${ }^{3}$.

It may be inferred from this evidence that the photochemical reaction between chlorophyll and ferrous sulphate does not take place according to the equation (1), and that it is not analogous to the photochemical reduction of the vat dyes. In view of the fact that this reaction takes place only in presence of oxygen, it may rather be regarded as a photo. chemical oxygen transfer, the ferrous sulphate acting as an acceptor. In this sense that reaction is in close analogy to the photochemical bleaching of chloro. phyll in presence of thiosinamine and other organic acceptors ${ }^{4}$ Photochemical reactions of this description take place also in the absence of acceptors, for example, in absence of ferrous sulphate, but with an incomparably smaller velocity. Further results on the photochemical oxidation of chlorophyll will be published elsewhere.

With respect to Weiss's experiments with the other dyes referred to in his note, I described more than four years ago identical experiments on the bleaching of thionine in presence of ferrous sulphate and sulphuric acid ${ }^{5}$. The photochemical reaction between methylene blue and ferrous sulphate has been studied, moreover, by K. M. Brandt ${ }^{8}$.

Physico-chemical Institute,

K. WEBER. Engineering Faculty,

University, Zagreb, Yugoslavia. March 16.

J. Weiss, NATURE, 138, 794 (1935).

"According to Willstätter and Stoll ("Assimilationsbuch", p. 226), chlorophyll is, moreover, destroyed by addition of acid.

${ }^{3}$ Cf. Willstatter and Stoll, 1.c., p. 415.

4 Cf. H. Gaffron and K. Wohl, Naturwiss., 24, 103 (1936).

s. Weber, Z. phys. Chem., B, 15, 38 (1931); Naturwiss., 28, 840 (1935).

K. M. Brandt, Arkiv för Kemi, 12, No. 7 (1935).

\section{Thermal Decomposition of Ethylene Oxide}

THe thermal decomposition of ethylene oxide vapour was first studied by Heckert and Mack ${ }^{1}$, who showed that the process has the essential characteristics of a kinetically unimolecular reaction. In many respects, however, their data were incomplete and the inter. pretations suggested rather unsatisfactory. We have recently made a detailed re-examination of the kinetics of the reaction. In view of recent discussion of the problem by several workers ${ }^{2}$, it may be desir. able to summarise now the results of our investigation, which although in general agreement with that of Heckert and Mack, is more complete.

We have measured the rate of the decomposition over the temperature range $435^{\circ}-505^{\circ} \mathrm{C}$. ; that is, up to temperatures $70^{\circ} \mathrm{C}$. higher than those used by Heckert and Mack, where the rate was very small. The use of the higher temperatures has both experi. mental and theoretical advantages. The products are predominantly carbon monoxide and methane, but hydrogen and ethane are also formed. The reaction is roughly 'first order over its course', but the velocity constant is noticeably dependent upon pressure, as found by Heckert and Mack. 